\title{
Transatlantica
}

Revue d'études américaines. American Studies Journal

\section{Visual and verbal self-portraiture / Se donner à voir, se donner à lire}

Aix-Marseille Université, 9 octobre 2020

\section{Sophie Vallas}

\section{OpenEdition}

\section{Journals}

Édition électronique

URL : https://journals.openedition.org/transatlantica/15881

DOI : 10.4000/transatlantica.15881

ISSN : $1765-2766$

Éditeur

Association française d'Etudes Américaines (AFEA)

\section{Référence électronique}

Sophie Vallas, «Visual and verbal self-portraiture / Se donner à voir, se donner à lire », Transatlantica

[En ligne], 1 | 2020, mis en ligne le 01 décembre 2020, consulté le 01 février 2023. URL : http://

journals.openedition.org/transatlantica/15881 ; DOI : https://doi.org/10.4000/transatlantica.15881

Ce document a été généré automatiquement le 1 février 2023.

\section{(c) (i) (9)}

Creative Commons - Attribution - Pas d'Utilisation Commerciale - Pas de Modification 4.0 International - CC BY-NC-ND 4.0

https://creativecommons.org/licenses/by-nc-nd/4.0/ 


\section{Visual and verbal self-portraiture / Se donner à voir, se donner à lire}

Aix-Marseille Université, 9 octobre 2020

\section{Sophie Vallas}

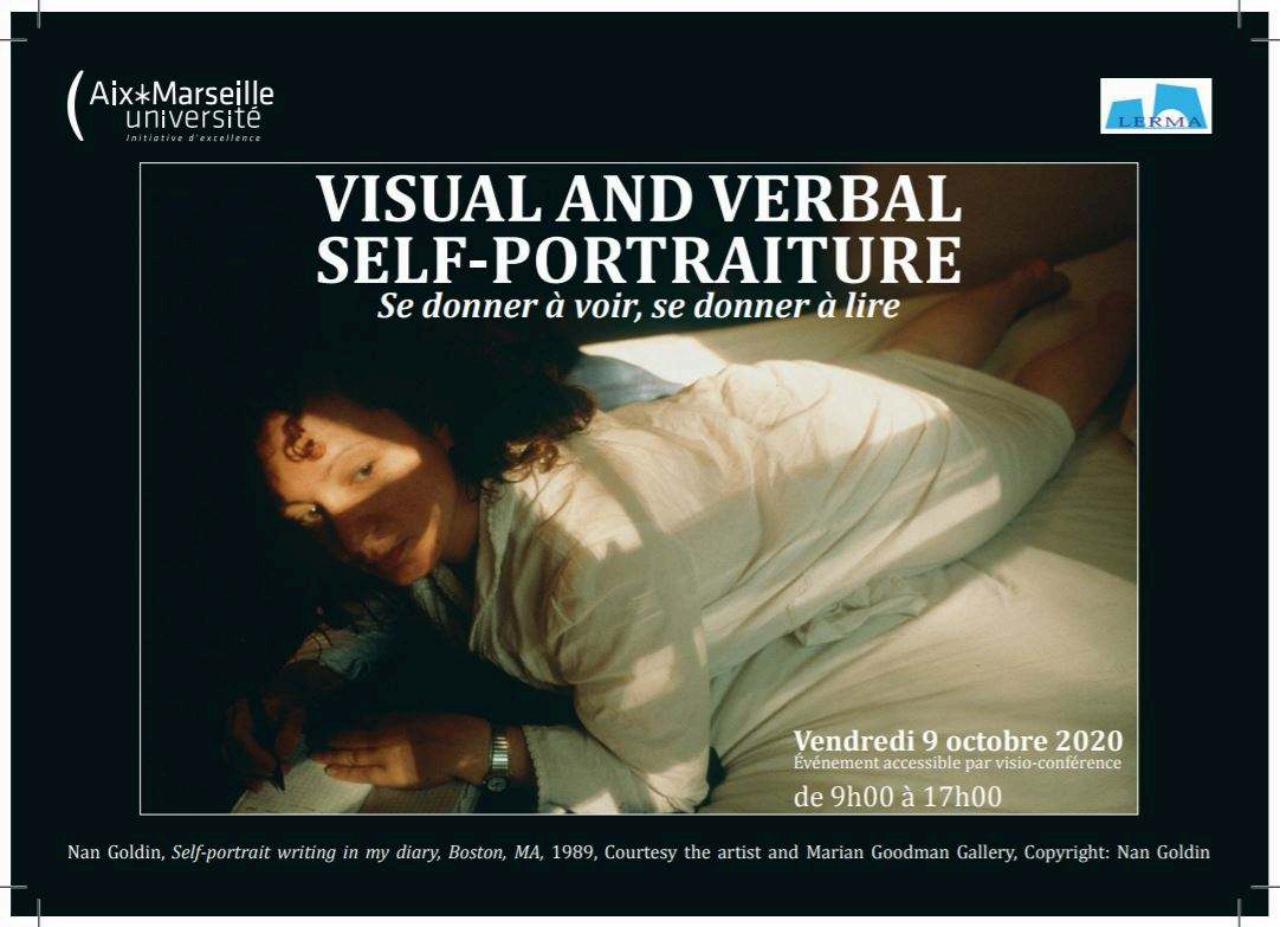

1 Comme son titre l'affichait clairement, la journée d'étude «Visual and verbal selfportraiture / Se donner à voir, se donner à lire ", organisée à distance par Jean-Louis Claret, Richard Phelan et Sophie Vallas (LERMA) à Aix-Marseille Université le 9 octobre 2020, portait sur l'art de l'autoportrait lorsqu'il est tout à la fois visuel et textuel. L'appel à communications rappelait les parallèles qui ont toujours été tracés entre la capture d'une image dans le miroir en verre du peintre et celle d'un reflet dans le «miroir d'encre» auquel Michel Beaujour, notamment, a consacré des travaux; il 
évoquait tout autant la longue pratique de l'autoreprésentation picturale comme jeu entre une image figée et l'histoire que toute toile contient, que la tradition de l'autoportrait littéraire, incarnée par Montaigne qui entendait se confronter à la tâche immense de se peindre avec des mots. Mais au-delà de ces traditions, la JE avait l'ambition de proposer une réflexion sur le travail d'autoportraitistes, écrivains ou artistes, qui incluent un dialogue manifeste entre le visuel et le scriptural dans leurs représentations d'eux-mêmes. À l'image de la photographie choisie pour illustrer l'événement, un autoportrait de Nan Goldin dans lequel la photographe, allongée sur un lit, est en train d'écrire, les communications ont toutes porté sur des œuvres qui mêlent l'image et le texte: autoportraits picturaux dans lesquels des textes, des inscriptions surgissent, ou autoportraits littéraires dans lesquels le sujet a inclus son visage griffonné, fidèlement ou non.

2 Danièle Berton-Charrière (Université de Clermont Ferrand) a ouvert la journée par une communication intitulée «Mary Stuart: from official pictorial and poetic portraiture to versified and embroidered self-depiction and assertion ». Muse ayant inspiré, en son temps, des auteurs, historiens et artistes qui ont fait son portrait de multiples façons, Mary Stuart fascinait tant par sa beauté, son intelligence, son érudition que par le pouvoir qu'elle incarnait. Berton-Charrière a analysé comment la reine déchue, une fois emprisonnée, déploya ses talents, déjà connus, de poétesse mais aussi de brodeuse pour réaliser un vaste autoportrait, à l'encre et à l'aiguille, qui lui permit tout à la fois de se montrer vulnérable, seule et désespérée, mais aussi de réaffirmer son identité et son innocence et de dessiner sa légende, dont elle devine la dimension politique et iconique.

Pour sa présentation intitulée "Nan Goldin: 'I'll be your (complex) mirror' ", Richard Phelan (Aix Marseille Université) avait emprunté l'un des titres du Velvet Underground and Nico ( I'll be your mirror») que Goldin elle-même avait choisi comme musique accompagnant The Ballad of Sexual Dependency, une projection de plusieurs centaines de photographies prises sur une dizaine d'années et dont la forme définitive fut exposée en Arles, en 1987. L'objet miroir, ainsi que le reflet qu'il permet, est au cœur du travail de Goldin qui pose comme double principe que l'autoreprésentation ne va pas sans la représentation de l'autre, et que la représentation de l'autre induit une représentation de soi-même : ainsi, Goldin elle-même ne figure pas sur toutes ces photographies qui, pour certaines, ne mettent en scène que des proches auxquels elle s'identifie de diverses façons et dont toute son œuvre souligne qu'ils font partie d'elle-même. Comme Phelan l'a subtilement démontré, Goldin est bien plus une artiste qui utilise la photographie qu'une photographe: diariste depuis l'âge de onze ans, une période marquée par le suicide de sa sœur, sa tâche est avant tout de noter des faits et des données, de conserver sa version des choses, d'enregistrer des voix et des messages (elle a gardé les cassettes de son répondeur depuis les années 1980). La photographie lui permet de pratiquer l'une des formes de l'auto-portrait, celle que permet l'écriture de la lumière, mais le cliché n'apparait jamais comme une fin en soi car Goldin, qui ne croit pas en son unicité, l'utilise comme un simple élément d'un récit à produire. «I don't believe in the single portrait. I believe only in the accumulation of portraits as a representation of a person ", dit-elle, et toute son œuvre montre en effet à quel point ses clichés sont conçus pour faire partie d'un tout, que ce soit un livre ou une exposition : ostensiblement pris sur le vif, sans mise en scène, souvent brouillons, de qualité moyenne, ils ne valent pas pour eux-mêmes mais pour leur capacité à faire mémoire, à tisser ce journal visuel que Goldin tient de sa vie dans ce qu'elle a de plus 
intime, de plus obscène parfois, et aussi de plus politique puisque le spectateur, en revisitant avec elle les années 80 , retraverse les années SIDA et l'utilisation politique de la sexualité par l'administration Reagan. Dans le débat qui a suivi cette présentation, plusieurs éléments centraux à la JE sont apparus, tels que la volonté de Goldin de réduire l'écart entre la vie et l'œuvre dans ce journal photographique qu'elle écrit quasiment au quotidien, une pratique qui évoque celle de Montaigne, par exemple, à travers la foi qu'elle place dans l'accumulation comme source d'un portrait infini et toujours recommencé, qui se construit par l'ajout. Enfin, la question de la frontière entre autoportrait et selfie est singulièrement posée par la pratique de Nan Goldin, qui repose beaucoup sur une prise d'image spontanée pourtant pas toujours compatible avec la préparation technique et la mise en scène que suppose l'autoportrait.

4 John Bak (Université de Lorraire), qui prépare un ouvrage sur les autoportraits de Tennessee Williams, a présenté un gros-plan sur l'année 1939, année charnière pour celui qui hésite, à l'époque, entre une carrière de dramaturge et une carrière d'artiste. Sa communication, intitulée "The Self as Other / the Other as Self: Tennessee Williams' Narcissistic Primitivism and Gauguin's Nave Nave Mahana», montre Williams en Californie, profondément marqué par des toiles de Gauguin, qui esquisse trois autoportraits picturaux dont un aux yeux bleux (sur lequel se concentre Bak) sous lequel il écrit : "Very flattering, even then ». L'été 1939 fut, dans les propres mots de Williams, le plus bel été de sa vie, au cours duquel il admet son homosexualité. Gauguin était son peintre préféré, celui qui avait célébré « les jours insousciants » (« nave nave mahana ») de sa période tahitienne. Comme son journal intime, publié posthumément, le met en lumière, le peintre vécut une véritable renaissance à Tahiti : la confrontation à l'autre, au sauvage, entraîne un nouveau regard sur lui-même, sur son identité la plus profonde, y compris sexuelle puisque l'on sait qu'il navigua alors entre les deux sexes dans une société qui acceptait cette ambivalence. Ses autoportraits le représentent en "sauvage primitif»- la peau beaucoup plus sombre, les traits empruntant à cet « autre » exotique - et redéfinissent ainsi la beauté comme une construction culturelle fondée sur des représentations occidentales genrées. Gauguin se lance alors dans une entreprise d'auto-mythologisation, la représentation du soi comme " autre » légitimant ses transgressions. Tennessee Williams marche sur les traces de Gauguin en 1939, et son auto-portrait donne forme à cet "autre » queer et primitif qu'il découvre et entend, d'emblée, célébrer comme son nouveau «moi»: des yeux bleus, un corps outrancièrement musclé et dont la peau sombre rappelle celle de Gauguin... Williams en est très conscient, qui commente cette prise de conscience dans sa correspondance. Le portrait de 1939 est la première pierre d'une démarche reposant sur un mythe narcissique emprunté à Gauguin et autorise Williams à se construire comme un objet de désir.

5 C'est une partie récemment rendue publique de la correspondance de Sylvia Plath que Laure de Nervaux-Gavoty (Université Paris-Est Créteil) a sondée dans une communication intitulée "'If only you could see me now': Autoportrait et théâtralité dans la correspondance de Sylvia Plath». Dans ses lettres à sa mère, Plath, prise au piège de la reconnaissance et de la culpabilité, cherche à transmettre à celle qui ne cessait de dire qu'elle s'était sacrifiée pour que sa fille puisse étudier une image idéalisée d'elle-même, en phase avec les attentes maternelles. D'une lettre à l'autre, qu'elle orne de dessins (dont plusieurs autoportraits) et poèmes, Plath trace un portrait d'elle-même qui puise aux clichés littéraires et cinématographiques des années 50 et qui lui permet d'apparaître comme la véritable héroïne d'une success story entièrement 
reconstruite ; l'expression «If only you could see me now... » est récurrente, insistant sur ces autoportraits verbaux qu'elle compose pour sa mère, choisissant soigneusement ses mots pour donner à voir l'image d'un bonheur conjugal sans égal alors même qu'il s'effondre; ces "photographies verbales " sont souvent redoublées par de véritables clichés insérés dans les lettres, dans lesquels Plath, même après son divorce, même au bord du suicide, continue à théâtraliser son moi et à dissimuler les fractures de sa vie (clichés de la jeune femme posant comme mannequin et publiés dans Varsity, ou clichés en famille). Nervaux-Gavoty a finement mis en lumière les paradoxes de cette correspondance dans laquelle Plath semble vouloir ne faire qu'une avec cette mère, réelle ou idéalisée, pour laquelle elle construit un autoportrait (le verbe "share", omniprésent, met l'accent sur la correspondance comme lieu d'échanges et de partage), tout en faisant entendre, in fine, une voix désespérément solitaire et fermée sur ellemême : l'écriture épistolaire de Plath, en fait, transforme l'autre en un simple miroir renvoyant au sujet une image figée et mortifère de lui-même.

6 «Soi-même comme un autre : James Agee et l'écriture documentaire de soi » a permis à Adriana Haben (Université Toulouse - Jean Jaurès) d'évoquer le roman d'inspiration autobiographique (A Death in the Family) que James Agee laissa inachevé à sa mort, en 1955. Publié dans une première version en 1957, il a fait l'objet d'une nouvelle publication en 2007, plus respectueuse des manuscrits d'Agee. Alors qu'Agee, dans des textes documentaires, dans des nouvelles ainsi que dans le célébré Let Us Now Praise Famous Men (publié en collaboration avec Walker Evans), avait fait entendre une voix autobiographique à la première personne, il écrivit $A$ Death in the Family, dont l'intrigue tourne autour de la mort de son père, à la troisième personne et prête au jeune héros son second prénom, Rufus, dont on sait qu'il ne l'aimait pas - le régime d'écriture de ce texte est donc complexe, entre roman autobiographique et autofiction. Si l'on sait qu'Agee avait pour projet d'inclure dans son roman des photographies ainsi que d'autres documents visuels (son travail comme critique de cinéma et scénariste pour Hollywood avait aiguisé chez lui un sens très sûr de l'image et du cadrage), ce qu'il n'a pas eu le temps de mettre en œuvre, Haben a montré avec précision à quel point l'écriture elle-même laisse une grande place au visuel : le texte, par exemple, accumule descriptions et portraits esquissés à travers les yeux enfantins de Rufus, rappelant en cela la tradition de la photographie de famille, très vernaculaire ; dans sa ponctuation, dans son découpage, il porte les traces d'un découpage et d'un montage empruntant aux techniques cinématographiques. À l'instar de tout son travail de journaliste et de documentariste, la fiction d'Agee vient explorer le réel dans ce qu'il a de plus quotidien, de plus modeste, de plus invisible parfois. Portrait du père mort trop tôt, autoportrait déguisé du fils, l'ouvrage inachevé conserve des contours flous, entre fictionnel et factuel, entre écriture et photographie.

7 Enfin, Jean-Marc Victor (Sorbonne Université) a clos cette JE avec une réflexion sur l'autoportrait en photographie intitulée «À la lettre (près): autoportraits de Ralph Eugene Meatyard». Rappelant tout d'abord que la pratique de l'autoportrait photographique suppose une mise en scène technique qui permet au sujet de redoubler sa présence, moi photographiant coexistant avec moi photographié, Victor ouvre ensuite l'univers fascinant de Meatyard, fait d'autoportraits incluant toujours des signes écrits (graffiti, journaux, inscriptions sur des murs, lettrages peints, plaques publicitaires...), comme autant d'amorces narratives, parfois à peine visibles, qui ne cessent pourtant de dialoguer avec l'image. Dans les photographies de Meatyard, le monde semble passé, décoloré, usé, en passe de s'effondrer ou de disparaître, et le sujet 
lui-même, simple élément ajouté à un ensemble hétérogène d'objets disparates, en est affecté. Photographe amateur, Meatyard s'est représenté seul ou entouré de sa famille, parfois masqué et dans d'étranges mises en scène. Il est difficile de rendre ici hommage aux analyses poussées des clichés de Meatyard, riches de tant des strates d'inscriptions à lire et à décoder dans leurs échos complexes, à la fois intertextuels et autobiographiques. Le débat qui a suivi a notamment rappelé l'omniprésence du signe écrit dans la culture américaine, et la tradition de la lettre dans la photographie aux États-Unis (voir Evans, par exemple).

Les échanges qui ont conclu la JE ont tout d'abord conforté les organisateurs : s'ils ne savaient guère quels types de propositions leur appel à communications, très pointu, allait recevoir, ils se sont déclarés ravis de la richesse des textes présentés. Certains échos entre les présentations ont été soulignés : tout d'abord, comme on pouvait s'y attendre, le caractère répétitif de l'autoportrait, souvent le travail d'une vie ou d'une partie importante d'une vie, travail patient, toujours recommencé, jouant subtilement sur le même et la variation, contrairement à l'autobiographie qui, elle, est davantage conçue comme une entreprise unique et récapitulative. L'autoportrait, au contraire, qu'il soit visuel ou scriptural et d'autant plus, dans le cas présent, lorsqu'il utilise les deux expressions, ne cesse de montrer à quel point le moi est diffracté, pluriel, changeant, et entraîne de ce fait ceux qui tentent de l'esquisser dans une quête inlassable et parfois déconcertante, remarquablement inventive en termes de mises en scène du moi et d'auto-mythologisation. Bifides, les autoportraits étudiés lors de cette JE engagent ainsi la sincérité des auteurs sans pour autant prétendre à une quelconque vérité. D'autre part, les communications ont également éclairé le recours, chez beaucoup de ces autoportraitistes, à une famille, réelle ou reconstituée, qui prend part à l'entreprise d'autoreprésentation et sert de substitut à l'identité propre de l'autoportraitiste: le passage par l'autre, par son regard bienveillant ou non, permet l'accès à un moi intime. Dans tous les cas, les échanges, directs ou codés, entre des visages et des textes, entre les reflets des autoportraitistes et ces lettres, mots, phrases qui les entourent, sont source d'une remarquable dynamique créatrice.

\section{INDEX}

Thèmes : Actualité de la recherche

\section{AUTEUR}

SOPHIE VALLAS

Aix-Marseille Université 